\title{
Una aproximación analítica a las mallas recíprocas diseñadas por Leonardo
}

\author{
Reciprocal frames designed by Leonardo. An analytical \\ approach
}

L. Sánchez $^{(*)}$, F. Escrigg ${ }^{(* *)}, M^{a}$ T. Rodríguez ${ }^{(* * *)}$

RESUMEN

Ya resulta tópico referirse a Leonardo cada vez que tocamos algún tema de arte, ciencia o técnica puesto que en sus escritos siempre podemos encontrar aspectos que nadie estudió antes ni, en muchos casos, nadie explotó después. Esto se explica porque sus manuscritos no fueron publicados hasta cinco siglos después. Sus observaciones respecto al diseño de estructuras son menos conocidas y son también las menos estudiadas. Realmente sus principales aportaciones se contienen en el Códice I de Madrid, y menos en el Códice II de Madrid, algunas otras, más generales, en el Códice Atlántico. Sus otros cuadernos de notas no tratan de estas cuestiones. En sus dibujos, Leonardo manifestó una obsesiva búsqueda de soluciones para cubrir grandes luces con piezas cortas. En sus Cuadernos encontramos diagramas de muchas variantes para construir cubiertas, forjados y puentes que pudieran salvar grandes luces con piezas manejables y disponibles. En este artículo se analizan con los actuales programas de cálculo cómo funcionan sus propuestas y establecemos algunas conclusiones.

403-21

Palabras clave: estructuras históricas, estructuras de rápido montaje, estructuras recíprocas, estructuras de madera, estructuras de acero.
SUMMARY

It is a topic to refer Leonardo when we introduce any branch of the art, science o technique because his manuscripts ever contain news that before him never where explored, nor profited even later. This can be understood because these manuscripts never where published till five centuries later. Observations referred to design of structures are the less known and otherwise the less studied. Really the most important problems about this subject are contained in the Codex I of Madrid, some in the Codex Atlantic, and less in the Codex II of Madrid. Other books do not refer these questions. Leonardo, in his drawings, manifested an obsessive search for solutions to the problem of how to span great distances with short pieces. In his notebooks, we find reiterative diagrams which seem to be searching for variants of frames capable of constituting structures, mainly roofs, floors and bridges, which could cover large distances by using manageable pieces that could easily be supplied. In this paper we analyze with actual computer programs how his proposals could be achieved and we establish some conclusions about it.

Keywords: historic structures, rapidly erected structures, reciprocal structures, wood structures, steel structures. 
1. Estudios para la construcción del cimborrio de la Catedral de Milán. Folio 310r-b del Código Atlántico.

2. Página 39 del Cuaderno de Villard de Honnecourt.

\section{DISEÑAR ESTRUCTURAS PARA SALVAR GRANDES LUCES CON PIEZAS CORTAS}

Leonardo, en sus dibujos, demostró una búsqueda obsesiva de soluciones para salvar grandes luces con piezas cortas. En sus cuadernos encontramos esquemas que reiteran y buscan variantes para mallas susceptibles de ser estructuras que puedan cubrir grandes superficies utilizando piezas manejables y de fácil provisión, principalmente techumbres, forjados y puentes. Probablemente él no pasó de dibujar sus invenciones y no las
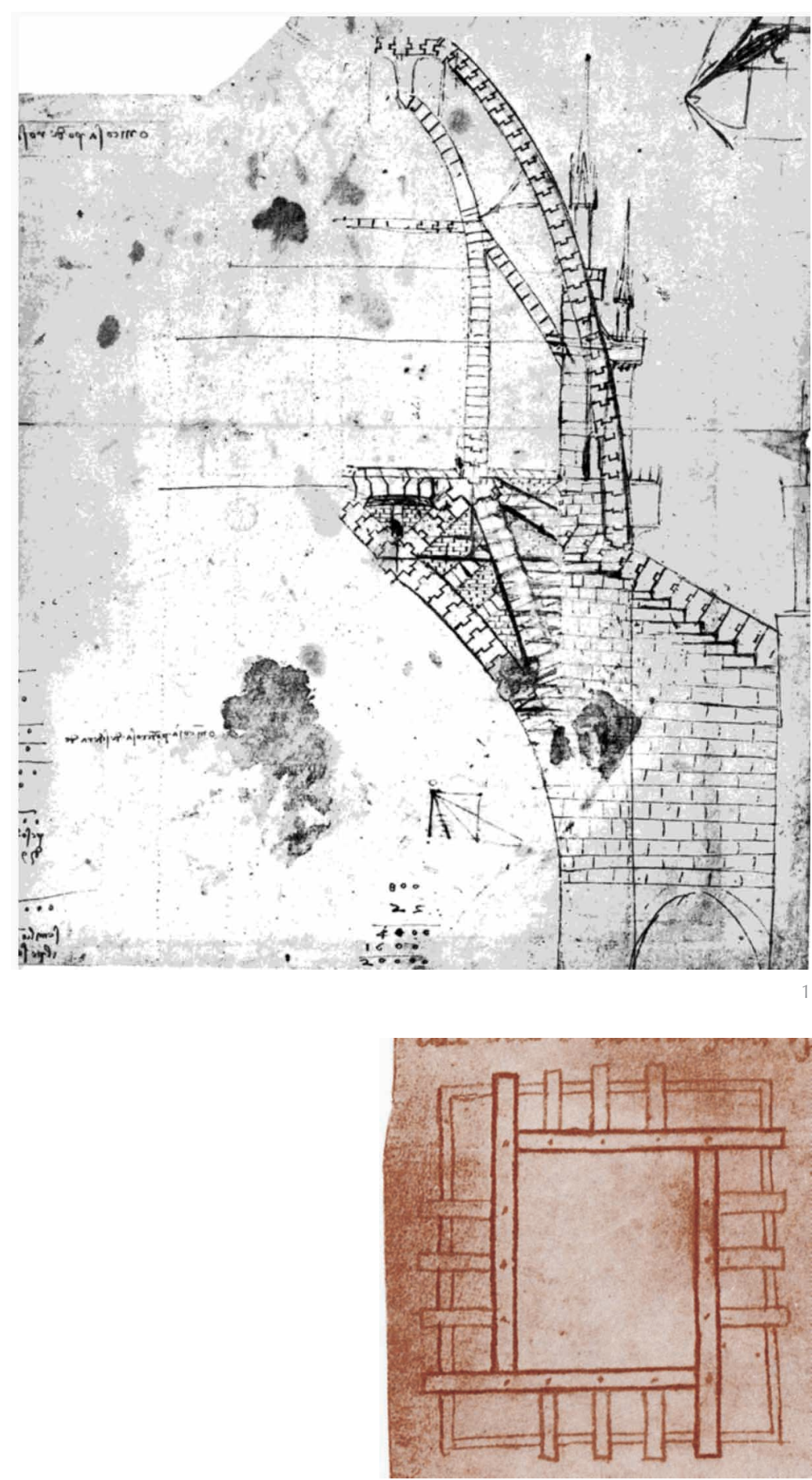

tanteó experimentalmente, pero resolvió todos los detalles como si realmente fuera a construirlas. Vamos a desarrollar algunas de estas aplicaciones a la luz de los conocimientos que tenemos ahora mismo. Es bien sabido que Leonardo conocía muchos inventos anteriores al respecto contenidos en numerosos tratados antiguos y contemporáneos (3). Trabajó en Siena, Milán y Florencia durante el periodo de sus grandes construcciones. Estuvo en contacto con la escuela sienesa de ingeniería cuyo máximo exponente, Giorgio Martini, escribió y publicó un tratado, "Trattato di Archittetura, Ingeniería y Arte Militar" (9), que difundió el estado del arte a través de magníficas láminas. También trabajó en el Duomo de Milán, y se conserva un ingenioso dibujo para levantar el cimborrio de su catedral (Figura 1) (7 y 10). También conocía los inventos de Brunelleschi para la construcción de la Cúpula de Santa María de las Flores que, aunque nunca fueron publicados, circulaban como material de trabajo en todos los gabinetes de proyecto. Además los "Diez Libros de Arquitectura" de Alberti eran una referencia obligada, poco detallada a nivel de soluciones concretas, pero muy sugerente a nivel general. Tal vez no conociera en particular otras referencias medievales como las de Villar de Honnecourd en su "Livre de portraiture" (15). Pero es sabido que sus soluciones ya formaban parte del repertorio de los arquitectos e ingenieros del momento (Figuras 2 y 3 ).

No es extraño por tanto que Leonardo hiciera variaciones sobre los temas que podía consultar y conocer y que no fuera absolutamente original a la hora de concebir sus soluciones. Su idea, en muchos casos era la de redibujar y mejorar. Se expresaba mucho mejor en los dibujos que en la ejecución, al menos en sus propuestas de ingeniería y arquitectura.

Tal vez no hayan llegado a nosotros los encargos directos que él trató de resolver en sus dibujos y que por tanto sus propuestas no fueran diletantes sino de aplicación práctica ya que sorprende la precisión y el detalle de las soluciones concretas. En cualquier caso, vamos a estudiar varios ejemplos en que aplicó esta capacidad inventiva.

\section{PUENTE PARA MONTARSE RÁPIDAMENTE CON TRONCOS SIN PREPARAR}

En el Códice Atlántico (Folio 22 recto) (7) podemos encontrar un puente construido con troncos con el que se supone que Leonardo pretendía dar una solución sencilla, económica y rápida para salvar obstáculos de pequeños ríos y cauces (Figura 4). Es una idea muy inteligente y eficaz basada en construcciones contemporáneas como las mostradas 


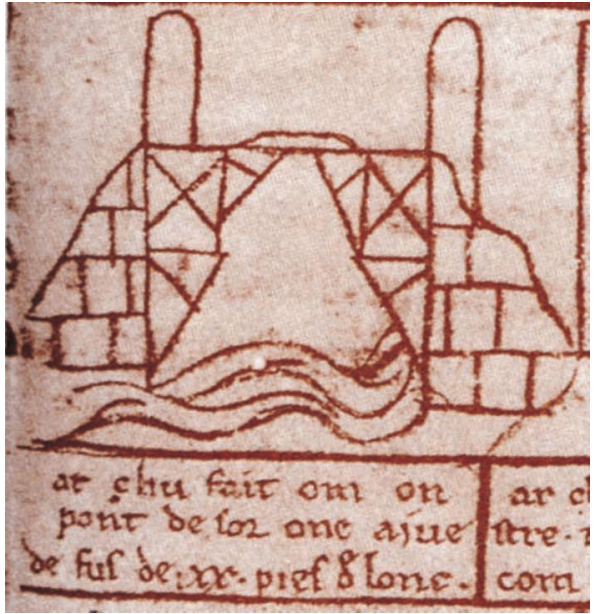

en la Figura 6, pero mucho más avanzada y definida gráficamente. Contamos con un precedente chino del siglo XII, el Puente del Arco Iris, construido realmente con esa misma idea (Figura 5) (16) y con muchas réplicas a través del tiempo hasta nuestros días. ¿Conocía Leonardo este precedente? Resulta difícil pensar que no, dada la enorme similitud de la solución aunque él hizo más hincapié en las soluciones constructivas que en la idea en sí, que parece admitir como ajena. Realmente lo complicado es saber cómo poner en pie ese conjunto de piezas de una forma ordenada y que funcione y eso es lo que precisa en varias secuencias de su dibujo de la Figura 4. En efecto, en la parte izquierda del dibujo y de arriba abajo, puede verse primero la alineación de troncos longitudinales y sus travesaños dispuestos en las embocaduras de las terminaciones antes de clavarlos en ellas. Todo este sistema plano está atado con cuerdas para poder empujarlo hacia el cauce y ser auxiliado con trípodes provisionales dentro de él. Luego viene el izado por tramos, y en cada izado se va empujando hacia el interior el tronco transversal de ese tramo. Tramo a tramo el puente cobra altura por sí mismo y, si se empieza desde el centro, se pueden ir retirando los trípodes provisionales de dentro a fuera. Sólo alguien que piensa en construirlo puede planteárselo de ese modo. En esta breve página hay contenida toda una lección de técnica y ningún esbozo es ocioso. La habilidad como dibujante queda patente en la facilidad con que deja clara constancia de las piezas que se cruzan y de las que pasan por delante o quedan de-

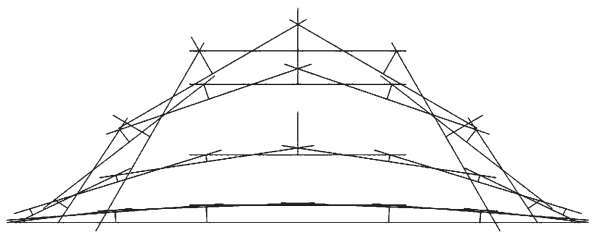

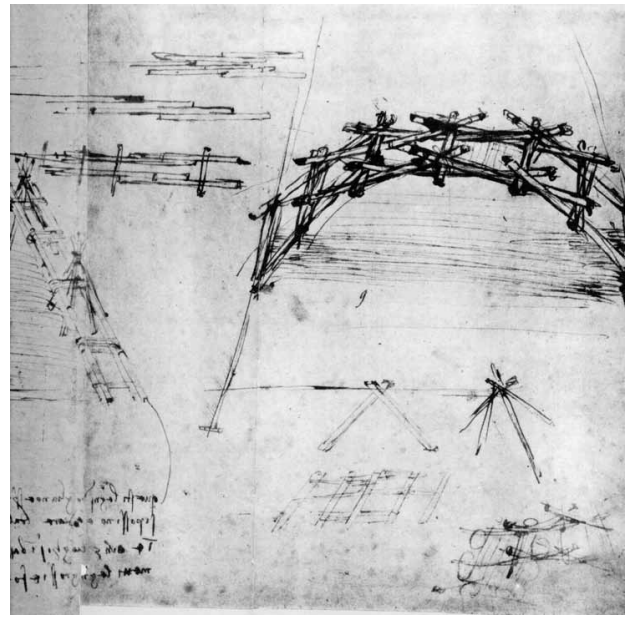

3. Página 45 del Cuaderno de Villard de Honnecourt.

4. Dibujo en el Folio 22 recto del Códice Atlántico.

5. Puente de la dinastía Song del Quingming Festival en el río Pien.

6. Proyecto Nova dirigido por Bashar Altagga para la televisión americana, que intenta recrear el Puente del Arco Iris. Utiliza elementos metálicos que no aparecieron ni en el original chino ni en la propuesta de Leonardo.

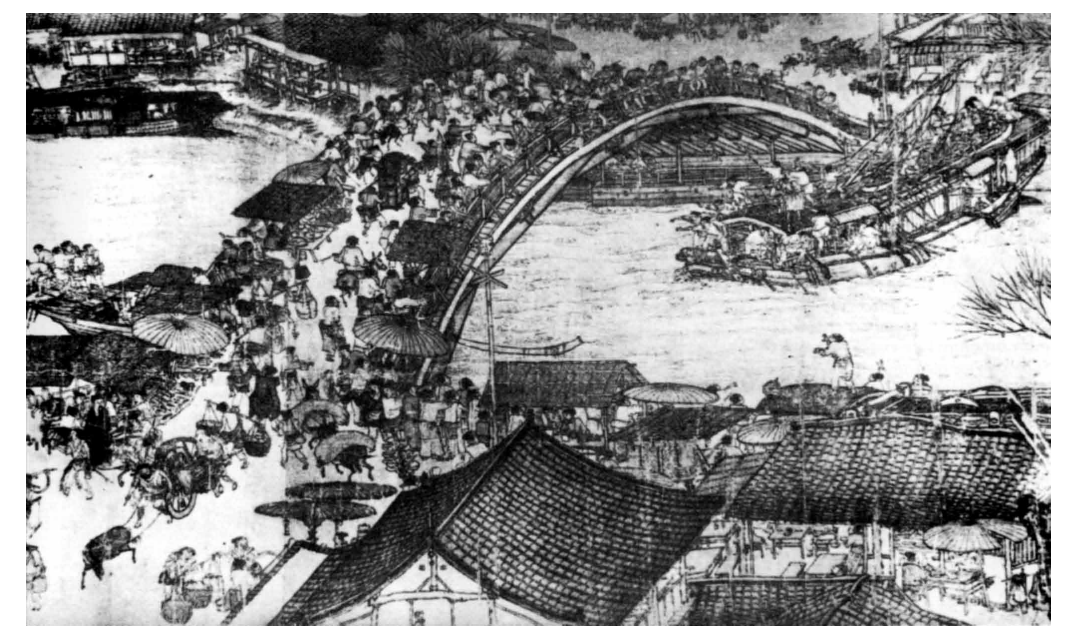

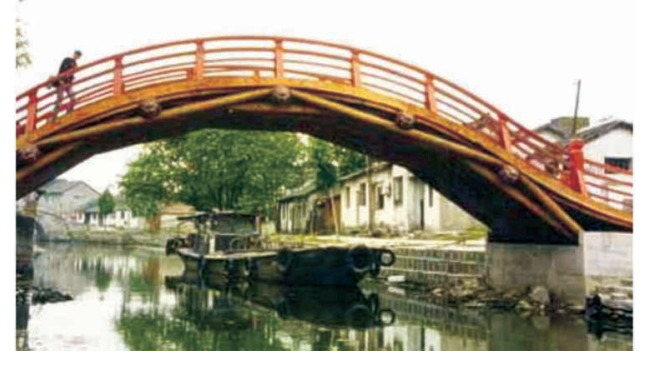

trás, sin que haya confusión, es más, parece quedar claro que no necesita ataduras una vez terminado el levantamiento y que, con un correcto estribado, ninguna pieza puede ya salir de su posición (Figura 7).

Los intentos recientes por reproducir el Puente de Leonardo o su precedente oriental (Figuras 4 y 5) no confiaron en la solución sin ataduras de Leonardo y han preferido asegurarse con tornillos en los cruces de barras (1).

Podemos pensar que Leonardo inventó el sistema sin referencias pero parece muy im-

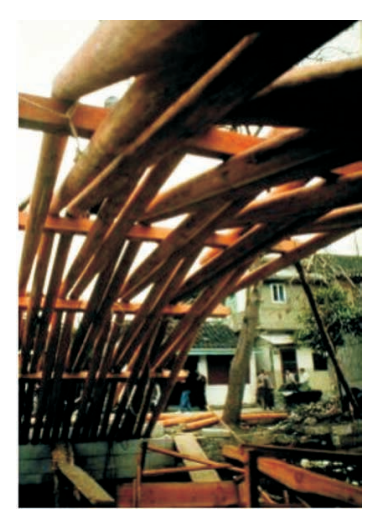

Distintos pasos para levantar el Puente de Leonardo. 


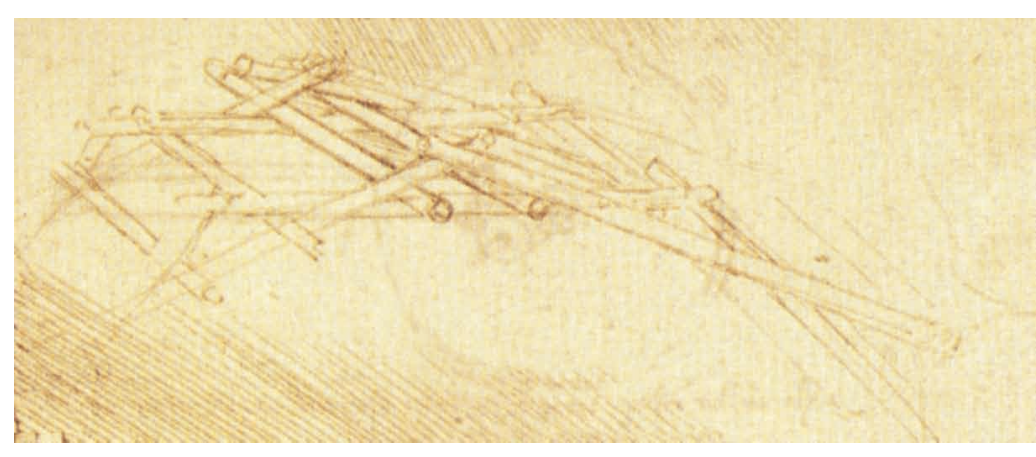

8
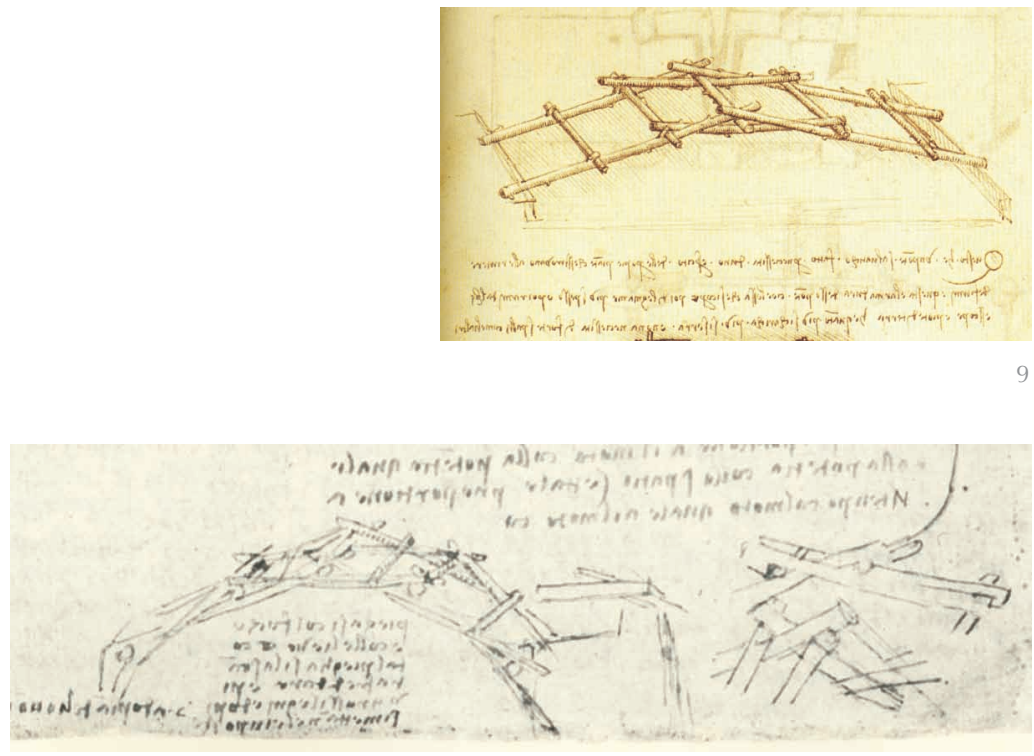

10

8. Puente dibujado en el Folio 45 recto del Códice de Madrid I.

9. Puente dibujado en el Folio 46 recto del Códice de Madrid I.

10. Puente dibujado en el Folio 183 recto del Códice de Atlántico.
El análisis que hemos planteado nosotros ha encontrado ciertas dificultades en la modelización de esas conexiones simplemente apoyadas y barras pasantes que sólo hemos podido solventar mediante un análisis de segundo orden para alcanzar soluciones coherentes ((5). Mediante el programa SAP 2000 hemos partido de la Figura 11 en donde los troncos transversales se han sustituido por barras de conexión articulada. En la Figura 12 podemos ver el esquema analítico con barras de $5 \mathrm{~m}$ de longitud y $40 \mathrm{~cm}$ de diámetro, lo que resulta geométricamente en una luz salvada de 14,50 m.

En la Figura 13 se muestra el esquema de momentos flectores debidos a peso propio con cargas aplicadas en nudos, mientras que en la Figura 14 se muestran los momentos flectores para una combinación de peso propio y cargas asimétricas de 2 t por nudo.

En la Figura 15 se muestra la deformada debida a esta última combinación.

Los extremos están articulados en ambos casos y el estudio que hemos hecho es plano. El puente está realizado con troncos de $5 \mathrm{~m}$ de longitud y $40 \mathrm{~cm}$ de grueso.

\section{CUBIERTAS Y FORJADOS}

Otro problema en el que Leonardo invirtió mucho esfuerzo es en el análisis de cubiertas y forjados resueltos con piezas cortas, más cortas que el vano a cubrir. Como en el caso anterior, ya Villard de Honnecourt (Figura 3) había representado en su cuaderno de notas soluciones para este problema. Esta solución en el futuro se llamaría "forjado serliano", a pesar de ser simplemente un autor de divulgación. Es realmente Leonardo quien plantea primero el problema con absoluta generalidad. Sus primeros esquemas son muy elementales y se basan en las soluciones conocidas (Figura 16).

Los investigadores y diseñadores actuales han acuñado el término estructuras recíprocas a éstas que se sostienen unas a otras en forma circular simple o generalizada (11).

Aunque la Figura 4 contiene el proyecto más desarrollado, Leonardo hizo numerosos esbozos en otros cuadernos hasta llegar a la solución. (Figuras 8 a 10) (7 y 8).

Las dificultades para resolver estas soluciones le hicieron preferir desarrollar con más detalle la propuesta de la Figura 4. Nunca hizo análisis del funcionamiento estructural, en parte porque no conocía mucho de cálculo vectorial y en parte porque ése no era el tema que más le preocupaba. En otras ocasiones sí se preocupó de estos aspectos analíticos (4, 12 y 13).
En la Figura 17 podemos ver un modelo realizado en madera y en la Figura 18 el análisis de los momentos flectores en $\mathrm{t} \times \mathrm{m}$ debidos al peso propio con barras de $6 \mathrm{~m}$ de longitud y secciones que se indican en el dibujo.

Desde un punto de vista estructural estos patrones dibujados por Leonardo parecen imposibles para su funcionamiento. Parecen demasiados cabalgamientos en falso para que la transmisión funcione. Pero los modelos físicos y analíticos confirman no sólo que funcionan, sino que lo hacen con gran efi- 

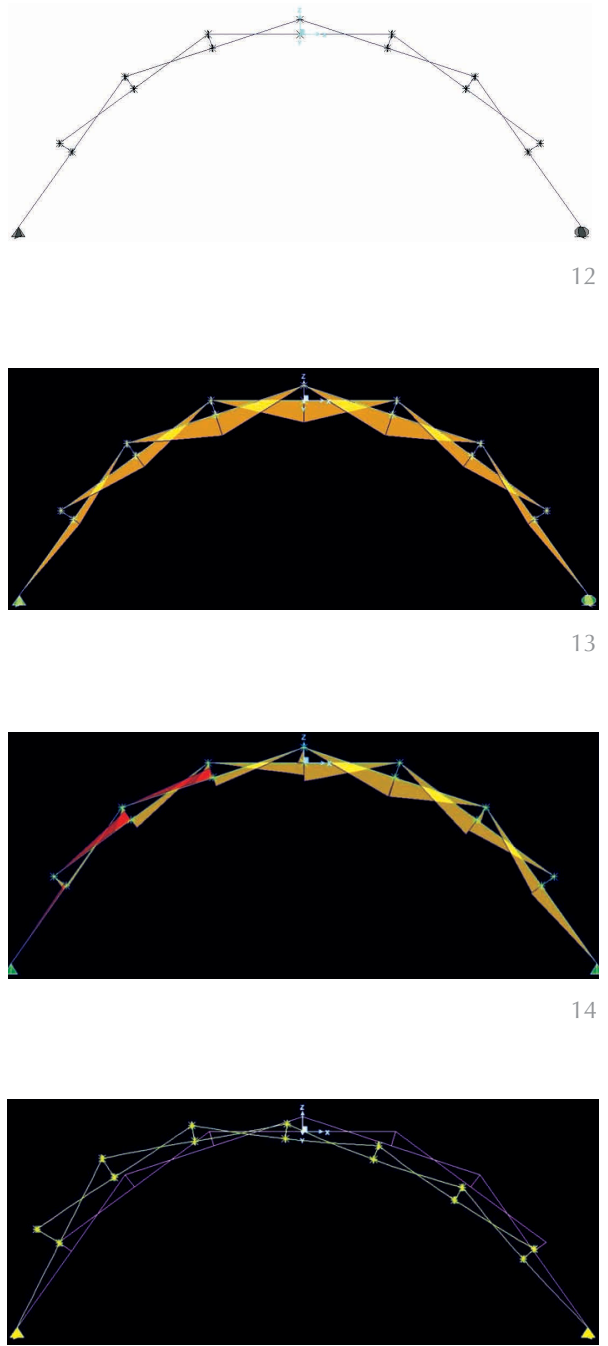

ciencia. En las siguientes páginas, las Figuras 20 y 21 muestran ejemplos construidos de los mismos.

En la Figura 20 vemos el modelo que desarrolla el dibujo A1 de la Figura 19. Se trata de dividir en tres partes la barra tipo y apoyar las piezas entre sí como se indica. Si hacemos en el extremo de las barras una muesca como la que se ve en la Figura 17 el desarrollo final será plano. Sin embargo, si las piezas apoyan considerando todo su grueso, la forma final se curvará en dos direcciones como se verá en un análisis que haremos más adelante.

En la Figura 21 se muestra otro patrón que Leonardo dibuja mucho menos nítidamente en el mismo folio a la izquierda del patrón señalado como B1 en la Figura 19. Es una teselación a base exclusivamente de cuadrados de dos tamaños diferentes.

El planteamiento analítico de las estructuras basadas en estos patrones se ha hecho con las simplificaciones necesarias para la definición geométrica. A saber:
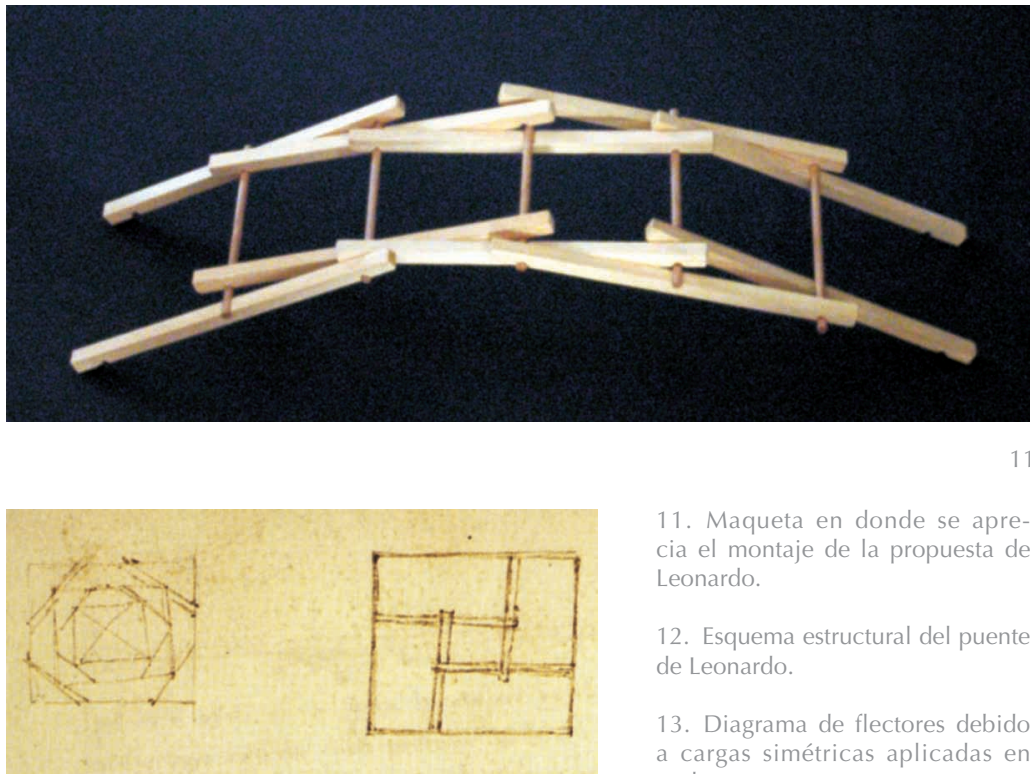

11. Maqueta en donde se aprecia el montaje de la propuesta de Leonardo.

12. Esquema estructural del puente de Leonardo.

13. Diagrama de flectores debido cargas simétricas aplicadas en nudos.

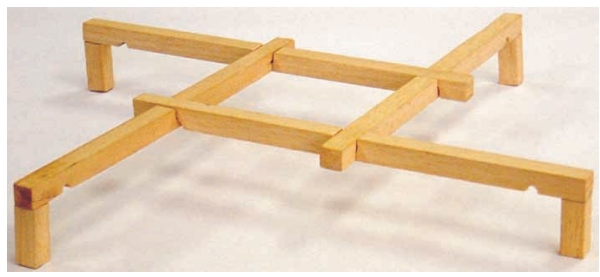

14. Diagrama de flectores debido cargas asimétricas aplicadas en nudos.

15. Diagrama de desplazamientos debidos a cargas asimétricas.

16. Dibujos en el Folio 49 verso del Códice de Madrid 1 .

17. El modelo realizado en madera del modelo de Leonardo.

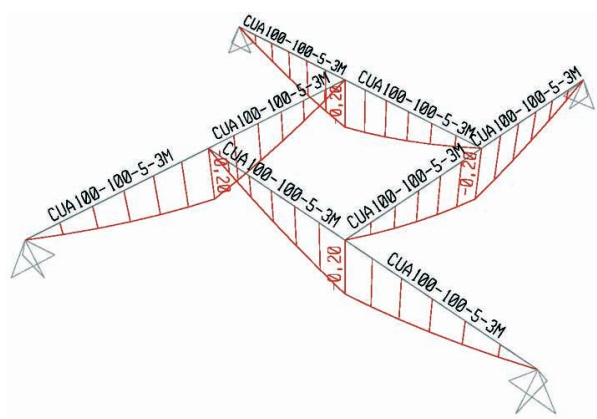

18. Análisis del funcionamiento del modelo de la Figura 17.

19. Códice Atlántico Fol 328 verso donde podemos ver distintos patrones de mallas recíprocas.

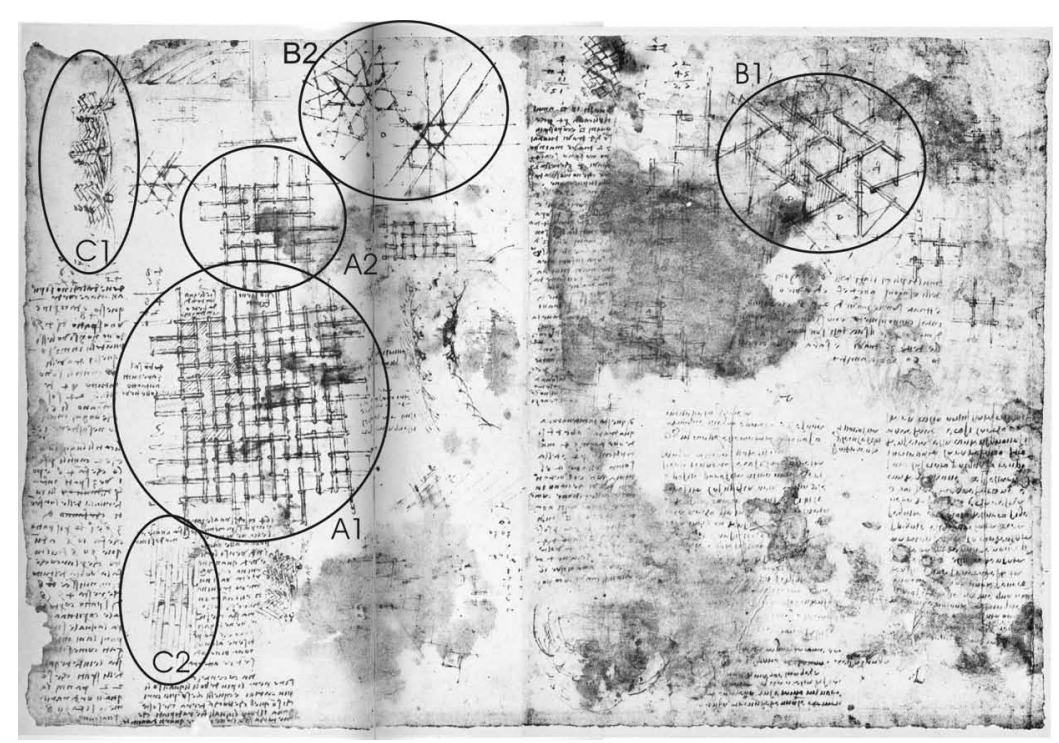


20 y 21. Modelos de los patrones dibujados por Leonardo en la Figura 19.

22 y 23. Modelo de la Figura 13 A1 y resultados de momentos flectores obtenidos por el Método de Elementos Finitos.

24 y 25 . Modelos para comparar los patrones rectangulares de Leonardo con un emparrillado convencional.

26 y 27 . Diagrama a escala de los momentos flectores representados en la Tabla I.

28. Modelo físico construido con el patrón de la Figura 19 B1

29. Resultados obtenidos en momentos flectores para el modelo plano con las mismas simplificaciones que hicimos en los modelos de las Figuras 20 y 21.
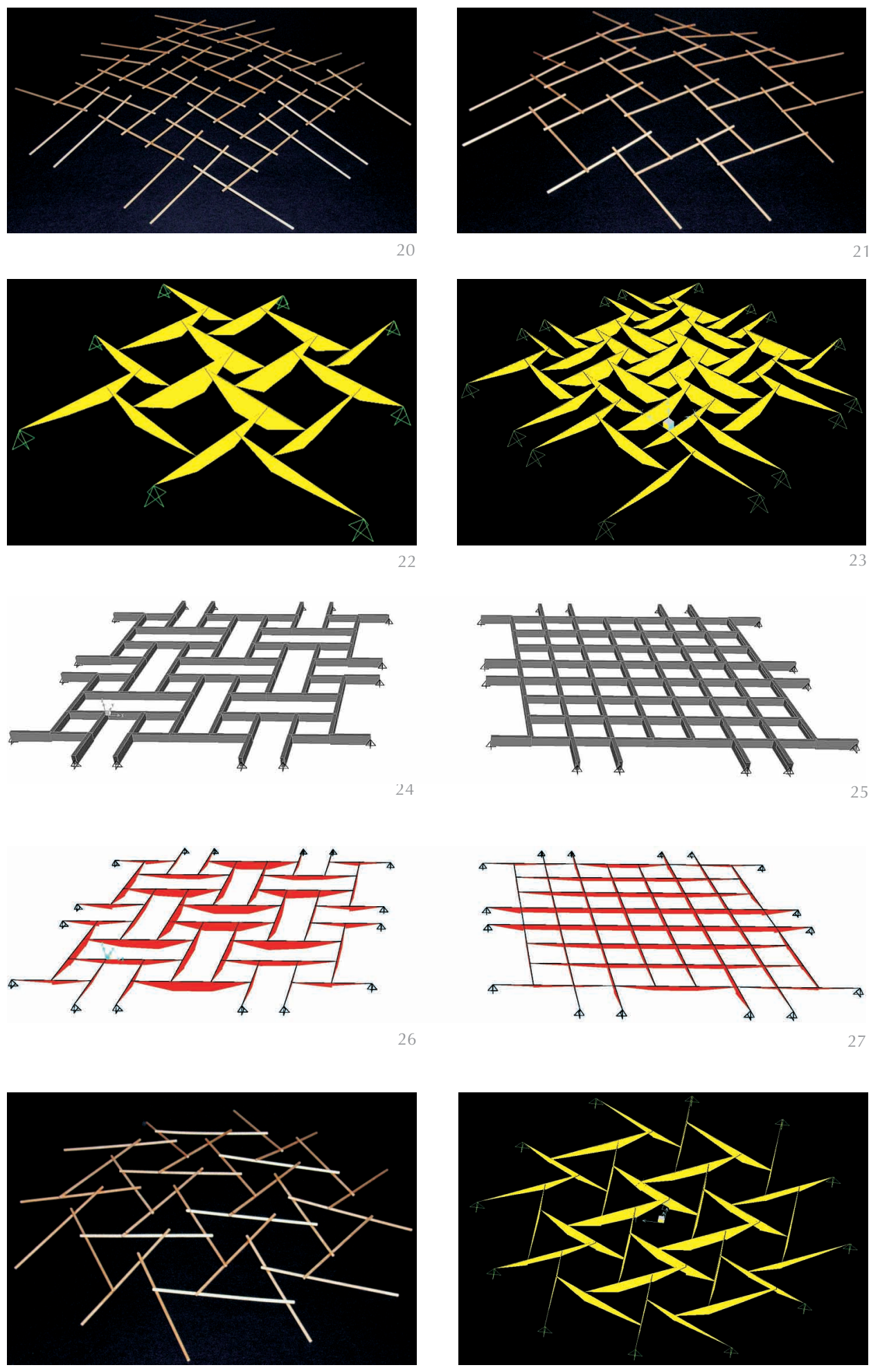

- Las barras tienen $3 \mathrm{~m}$ de longitud y los puntos de apoyo se señalan a $1 \mathrm{~m}$.

- Consideramos las acciones de peso propio aplicadas en los nudos.

- Los apoyos de unas barras en otras son articulados.

-El modelo es, por tanto, plano.

En las Figuras 22 y 23 se muestra el esquema de los momentos flectores resultantes consi- derando exclusivamente el peso propio para los patrones de las Figuras 20 y 21.

Si comparamos los resultados de un forjado cional representado en la Figura 25, con las mismas dimensiones globales y pesos totales obtenemos los resultados reflejados en la Tabla I. En momentos flectores la diferencia es del $30 \%$ mientras que en flechas es del $25 \%$ como el de la Figura 24 con uno conven- 
Tabla I

Comparación entre el comportamiento de las estructuras de las Figuras 24 y 25

\begin{tabular}{|c|c|c|c|c|c|c|}
\hline TABLA I: Figuras 24 y 25 & $9 \times 9 \mathrm{~m}^{2}$ & & & Peso propio & PP+Sobrecarga & $\begin{array}{c}\text { Momento } \\
\text { flector }\end{array}$ \\
\hline & Long. total & Peso total & Espesor & Flecha & $200 \mathrm{~kg} / \mathrm{m}^{2}$ & Ton $\times \mathrm{m}$ \\
\hline $\begin{array}{c}\text { Figura } 24 \\
\text { \#200X100 e.6 mm }\end{array}$ & $\mathrm{m}$ & Ton & $\mathrm{mm}$ & $\mathrm{m}$ & $\mathrm{m}$ & 2,8 \\
\hline $\begin{array}{c}\text { Figura 25 } \\
\text { \#200X100 e.4.66 mm }\end{array}$ & 112 & 2,3871 & 6 & 0,01 & 0,07 & \\
\hline
\end{tabular}

Tabla II

Comparación entre el comportamiento de las estructuras de las Figuras 30 y 31

\begin{tabular}{|c|c|c|c|c|c|c|}
\hline TABLA II: Figuras 30 y 31 & Barras 3 m & & & Peso propio & PP+Sobrecarga & $\begin{array}{c}\text { Momento } \\
\text { flector }\end{array}$ \\
\hline & Long. total & Peso total & Espesor & Flecha & $200 \mathrm{~kg} / \mathrm{m}^{2}$ & Ton $\times \mathrm{m}$ \\
\hline $\begin{array}{c}\text { Figura 30 } \\
\text { \#200X100 e.6 mm }\end{array}$ & $\mathrm{m}$ & Ton & $\mathrm{mm}$ & $\mathrm{m}$ & $\mathrm{m}$ & \\
\hline $\begin{array}{c}\text { Figura 31 } \\
\text { \#200X100 e.3.105 mm }\end{array}$ & 174 & 2,8652 & 6 & 0,0068 & 0,061 & 4,6 \\
\hline
\end{tabular}

para unos emparrillados que, en conjunto, pesan lo mismo.

En cuanto al patrón obtenido con mallas triangulares (Figura 19 B1) las conclusiones obtenidas son parecidas. En la Figura 28 vemos un modelo construido y en la Figura 29 una distribución a escala de los momentos flectores debidos a carga en nudos para un modelo plano.

Si comparamos los resultados de un forjado como el de la Figura 30 con un emparrillado triangular convencional representado en la Figura 31, con las mismas dimensiones globales y pesos totales obtenemos los resultados reflejados en la Tabla II. En momentos flectores la diferencia es del $46 \%$ mientras que en flechas es del 30\% para unos emparrillados que, en conjunto pesan lo mismo. En este caso los resultados de la disposición en hexágonos es menos eficaz que la disposición en rectángulos, para el mismo peso total y para una estructura plana.

Las Figuras 32 y 33 muestran la comparación de momentos flectores de los dos casos de la Tabla II representados a escala.

\section{CONSIDERACIONES SOBRE LAS BARRAS CON ESPESOR}

Si consideramos que las piezas no están conectadas a eje sino que se apoyan a cara superior y que, por tanto, toman la forma correspondiente a una superficie curva, tal como se aprecia en el modelo de la Figura 21 el modelo analítico cambia y los resultados también lo hacen de una manera radical, sobre todo si articulamos los nudos de borde y no les permitimos el desplazamiento.

Si comparamos los resultados de un modelo espacial (Figura 34, pág. siguiente) como éste con uno plano (Figura 35, pág. siguiente) encontramos grandes diferencias, ahora a favor del modelo de Leonardo, ya que se introducen axiles en barras para la transmisión de fuerzas.

En las Figuras 36 y 37, pág. siguiente, podemos ver, comparativamente la diferencia en los valores de los momentos flectores. Mientras que en la primera el momento flector máximo es de 0,29 t x m, en la segunda
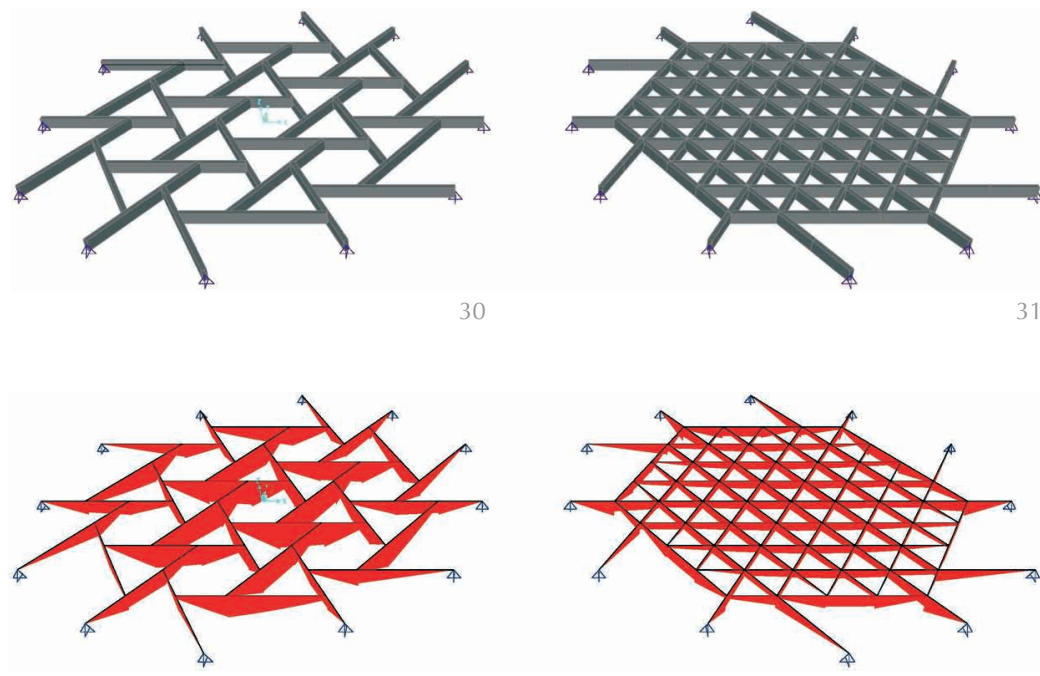
34. Modelo curvo, barras apoyadas a cara superior.

35. Modelo plano, barras conectadas a eje.

36. Momentos flectores para el modelo espacial de Leonardo.

37. Momentos flectores para el modelo plano. llegamos a 3,17 $\mathrm{t} \times \mathrm{m}$ para peso propio. Estamos considerando barras del mismo peso en ambos casos. Las acciones consideradas son $150 \mathrm{~kg} / \mathrm{ml}$ por barra aplicada como carga continua.

En la Figura 38 vemos el resultado de los esfuerzos axiles para el modelo espacial y no se compara con el modelo plano porque éste no los genera.

\section{OTROS MODELOS DE LEONARDO}

En el mismo dibujo de la Figura 19 y en los esbozos señalados como C1 y C2 Leonardo propone ejecutar una plataforma de gran rigidez basada en los mismos principios que sus puentes pero con barras curvadas. Esta plataforma está construida en el modelo de la Figura 39 y, aunque parece de una geometría elemental tiene una cierta complejidad que se ilustra en la Figura 40.
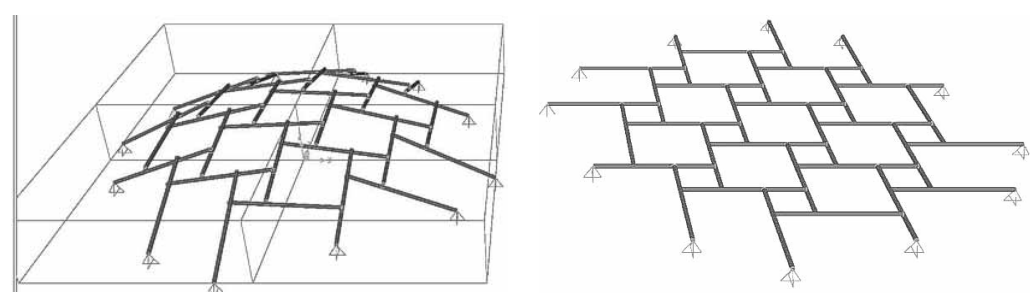

34

35
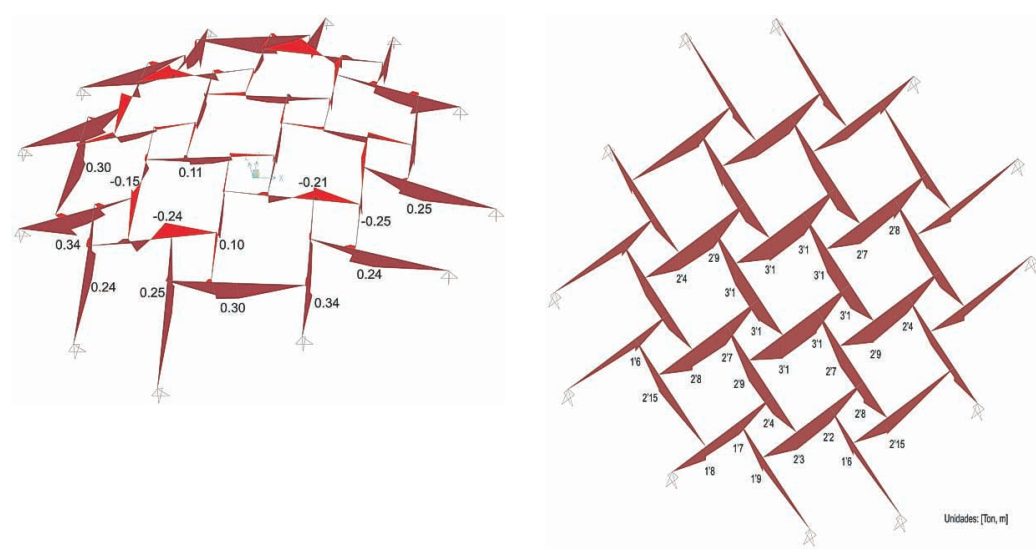

36

La pregunta, al igual que en los otros casos vistos anteriormente es si esta construcción tiene eficacia estructural en comparación con los diseños convencionales que se le aproximan en forma. Para ello planteamos la comparación adecuada.

Sea la estructura de la Figura 41 la que puede asimilarse a un modelo plano equivalente y vemos en la Figura 42 dos modelos analíticos, el A referido a una cercha triangulada convencional y el B a la propuesta de Leonardo. En ambos modelos el aspa significa nudo rígido y el círculo articulación. Las ba- rras que se cruzan sin símbolo son pasantes. Nótese que en algunos nudos hay barras que pasan continuas y otras que se articulan en ese nudo.

El cálculo que hemos realizado para establecer la comparación se ilustra en la Tabla III y las Figuras 43 y 44, página 14. Las secciones de barra consideradas son \#100x100 e.6 y las cargas son las de la hipótesis de peso propio y la de $200 \mathrm{~kg}$ verticales en cada nudo. Un extremo de la viga tiene los apoyos deslizantes. La longitud total de las cerchas es de $7,5 \mathrm{~m}$.

Como se aprecia en la Tabla III los desplazamientos de la cercha de Leonardo son un $58 \%$ superiores a los de una viga convencional con respecto a los desplazamientos pero desarrolla unos esfuerzos axiles parecidos (Figura 43). Los momentos también son parecidos en la zona central, que es la que realmente sirve de comparación (Figura 44).

Las plataformas de las Figuras 39 y 40, resueltas con barras curvas cortas se comportan, como hemos visto con una gran eficacia y son un modelo útil para aplicaciones estructurales.

\section{CONCLUSIONES}

Todos los patrones estudiados en el artículo están extraídos de dibujos de Leonardo y llama la atención que, pese a los numerosos estudios que recientemente han desarrollado los conceptos de reciprocidad o cabalgamiento, son muy pocas las aplicaciones prácticas.

En algunas de nuestras aplicaciones arquitectónicas hemos hecho usos de estos conocimientos de Leonardo para definir estructuras modulares, es decir, conjuntos realizados con piezas pequeñas para constituir grandes conjuntos. Vayan como ejemplo los contenidos en (6). (Figuras 45 y 46, página 14). Hemos intentado justificar que las aplicaciones estructurales son posibles y que la versatilidad es inmensa. Para ampliar esta información desde el punto de vista conceptual (2).

Tal vez Leonardo fue un investigador frustrado que apenas construyó ninguno de sus inventos aunque esta afirmación resulta dudosa a la vista de la perfección con que trabajaba los detalles en los dibujos. Pero lo cierto es que sus trabajos han estado perdidos hasta principio de los años 50 del siglo pasado y es ahora cuando podemos aprovechar muchos de sus conocimientos.

Éste es el objetivo principal del artículo $(3,14)$. 
Tabla III

Desplazamientos comparativos en las vigas de la Figura 42

\begin{tabular}{|l|c|c|c|c|c|}
\hline \multirow{3}{*}{ TABLA III } & \multicolumn{5}{|c|}{ COMPARACION ENTRE UNA CERCHA CONVENCIONAL Y LA DE LEONARDO } \\
\cline { 2 - 6 } & DESP. VERTICAL & DESP. VERTICAL & $\begin{array}{c}\text { DESP. } \\
\text { HORIZONT }\end{array}$ & REACCIONES & REACCIONES \\
\cline { 2 - 6 } & $\mathrm{PP}$ & COMB & COMB & VERTICAL-IZQ. & VERTICAL-DER. \\
\hline & $\mathrm{mm}$ & $\mathrm{mm}$ & $\mathrm{mm}$ & Ton & Ton \\
\hline CONVENCIONAL & $-2,8$ & $-14,1$ & $+1,6-1,6$ & 0,47 & 0,36 \\
\hline LEONARDO & $-4,4$ & $-22,4$ & $+2,6-2,6$ & 0,47 & 0,36 \\
\hline
\end{tabular}

Sob. $=200 \mathrm{~kg}($ por nudo $)=4 \times 200$

sob.
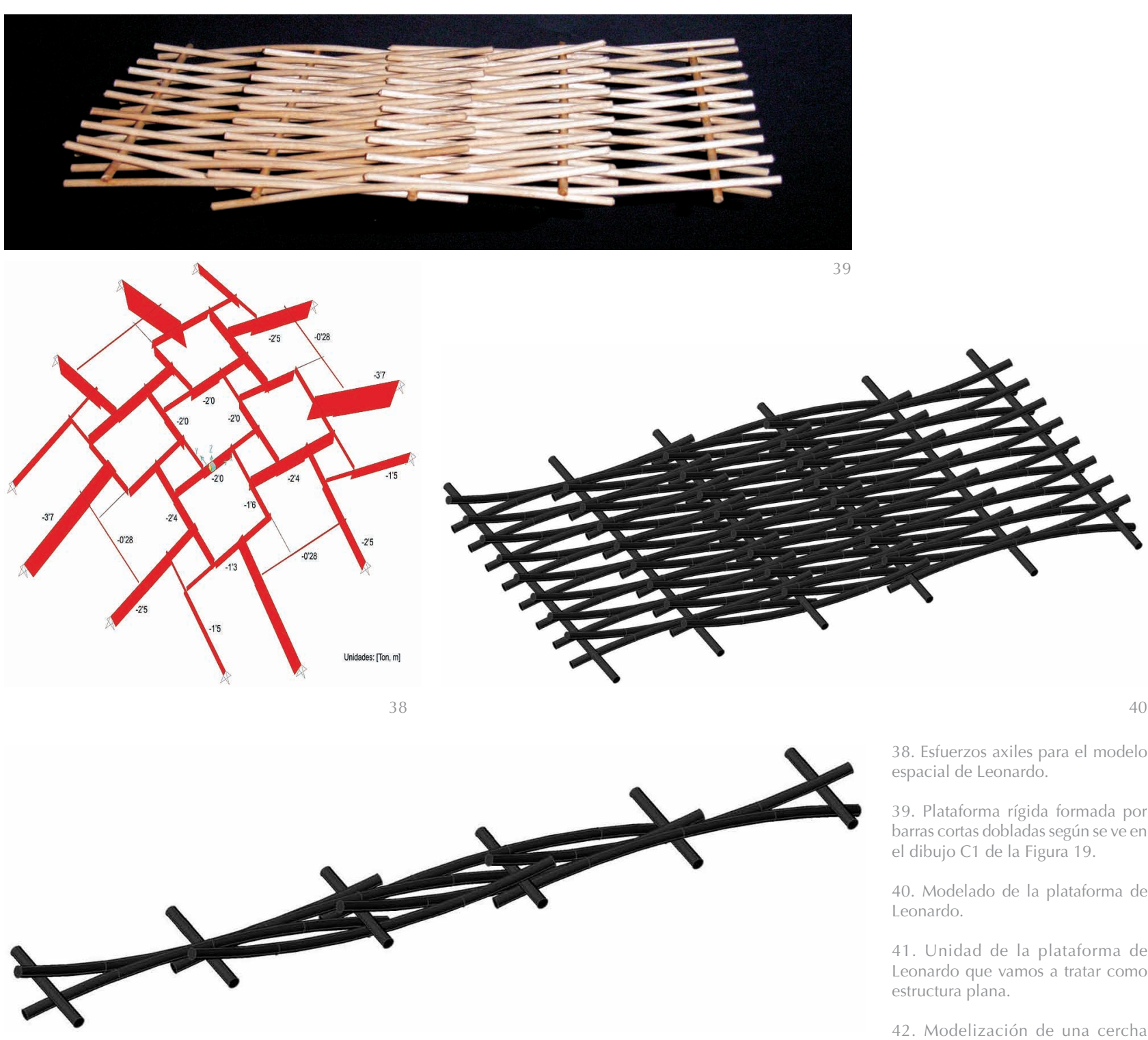

38. Esfuerzos axiles para el modelo espacial de Leonardo.

39. Plataforma rígida formada por barras cortas dobladas según se ve en el dibujo C1 de la Figura 19.

40. Modelado de la plataforma de Leonardo.

41. Unidad de la plataforma de Leonardo que vamos a tratar como estructura plana.

42. Modelización de una cercha triangulada (A) y la cercha de la plataforma de Leonardo (B).

A

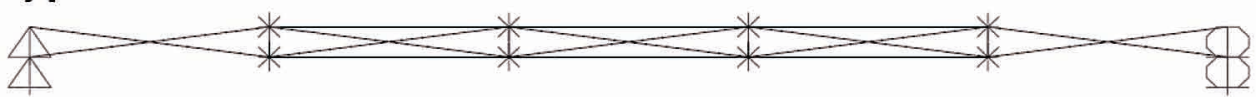

B

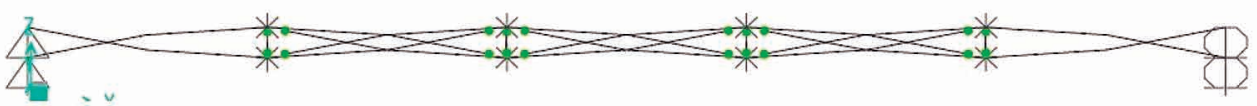



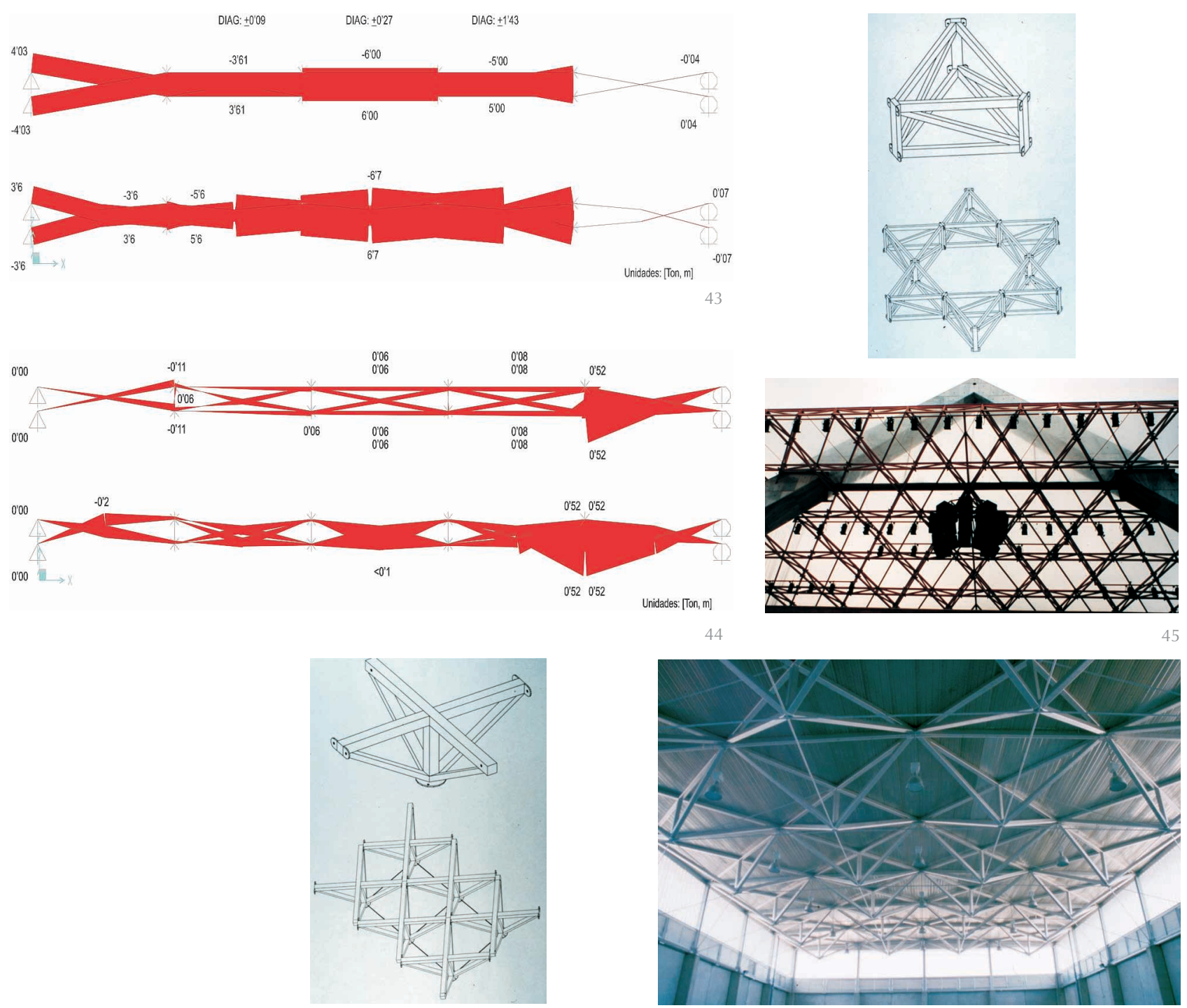

46

\section{REFERENCIAS}

43. Esfuerzos axiles en ambos casos.

44. Momentos flectores en ambos casos

45. Sistema modular con el que se construyó el tornavoz del escenario del Auditorio de la Rábida en Huelva por Escrig y Álvarez en 1988. Es una degeneración de la propuesta de la Figura 31

46. Sistema modular con el que se construyó el Pabellón de entrenamientos en el complejo deportivo de Huelva por Escrig y Álvarez en 1998 y antes en el Pabellón de Extremadura de la Expo 92 de Sevilla de Escrig y Curbelo. Es una degeneración de la propuesta de la Figura 25.
(1) Bashar Altabba P. E.: "Re-creating the Rainbow Bridge" ASCE Civil Engineering n. 5. May 2000. http://www.pbs. org/wgbh/nova/lostempires/china/builds.html.

(2) Baverel, O., Nooshin, H.: Nexorades Based on Regular Polyedra. Nexus Network Journal 2007. Kim Williams Books, Turin. 2007. pp. 281-298 ISSN 1522-4600.

(3) Duhem, P.: "Etudes sur Léonard de Vinci. Ceux qu'il a lus et ceux qui l'ont lu" Librairie Scientifique A. Hermann. París 1906.

(4) Escrig, F., Sánchez, J.: La geometría de las estructuras I. STAR 12. Sevilla. 2005

(5) Escrig, F., Sánchez, J.: Adaptable Leonardo. International Conference on Adaptable Building Structures. Eindhoven. July 2006. ISBN 90-72152-03-4. pp. 5.28 a 5.32.

(6) Escrig, F., P. Valcárcel, J.: "Modular Space Frame Structures". Space Estructures. Ed. Parker. Thomas Telford, London 1993. ISBN: 07277-1968-8.

(7) Leonardo Da Vinci. Códice Atlantico. Biblioteca Ambrosiana. Milán.

(8) Leonardo Da Vinci. Códice Madrid I y II. Biblioteca Nacional. Madrid.

(9) Martini, F. di Giorgio: "Trattati di Architettura Ingegneria e Arte Militare" Milano II Polifilo, 1967.

(10) Pedretti, C.: Leonardo architetto. Electa. Milan 1988. ISBN 8843525190.

(11) Popovich, O.: "Reciprocal Frame Architecture" Architectural Press. Amsterdam 2008. ISBN 978-0-7506-8263-3.

(12) P. Valcarcel, J. Escrig, F. "Pioneering in expandable structures: The Madrid I Notebook by Leonardo da Vinci". Bulletin of the IASS. Vol. 35. April 1994, n1 114. pp. 33-45. Madrid. ISSN 0304-3622.

(13) P. Valcárcel, J; Escrig, F.: “Un primer planteamiento de estructuras desplegables. el Código I de Madrid de Leonardo da Vinci". Boletín Académico de la ETSA, n1 10. La Coruña, 1989. pp. 13-20. ISSN 0213-3474.

(14) Truesdell, C.: Ensayos de Historia de la Mecánica. Ed. Tecnos 1975. ISBN 84-309-05979. pp. 17 a 88. Madrid.

(15) Villard de Honnecourt.: "Cuaderno" Ed. Akal 1991. ISBN 84-7600-670-5. Madrid

(16) WWW.: "Rollo del museo de Beijing" http://www.huangshantour.com/english/river.htm. 